\title{
Semantic employment of color in Syrian drama: Bab Al-hara movie as a model
}

\author{
Dr. Adnan Kazem Amansef, Ministry of Education, General Directorate for Vocational Education, \\ Hha_trap@hotmail.com
}

\begin{abstract}
Color is one of the important topics in the art world, especially in cinema and television. It is a cornerstone of the creative process. Therefore, this topic is given a great deal of importance in the present study. Chapter one includes a study of the methodological framework in terms of studying the research problem, its significance, objectives, limits, and the definition of terms. Chapter two tackles the theoretical framework. It is divided into three sections. Section one discusses significance as a term and a concept. Section two tackles color in the TV. Section three presents significance and meaning in visual implementation of color. Through the theoretical framework, the researcher extracted a set of indicators. The research procedures, the research method, the sample selection and its justifications, the research tool, and the unit of analysis are discussed in chapter three. Chapter four is devoted to the sample analysis. Chapter five includes the final results, conclusions, recommendations, suggestions, and a list of references and appendices.
\end{abstract}

Keywords: Significance, color, drama.

Received: 02.12.2020 $\quad$ Accepted: 19.01.2021 $\quad$ Published: 06.02.2021

\section{Chapter one}

The systematic framework.

\section{The research problem}

Color is one of the important topics in the world of art in general and in cinema and television in particular. It is related to the relationship between the recipient and the recipient of the film. International cinema could employ color as a sign in many of its works. Arab drama, whether cinematic or television, such as Egyptian, Algerian, Syrian, Gulf, or other Arab countries whose film or television production. Color connotations emerge from the aesthetic and dramatic recruitment process according to directive processing. The researcher identifies the problem in the following question:

What are the ways through which the color is employed to produce the significance in TV drama?

\section{The significance of the present study}

The significance of the present study comes from the fact that it is a specialized study in the department of audio and visual arts in an important subject in the field of color and its semantic investments in TV drama. In addition, it provides the specialized library with a new study. Besides, the study is theoretically and practically important for students of the Institute and the Faculty of Fine Arts. Workers in Satellite stations and workers in the jurisdiction of TV drama benefit from the present study.

\section{The Objectives of the present study}

The present study is an attempt to find out how color is semantically employed in TV drama.

The Limits.

The present study is limited to the Syrian TV drama; Bab Al-Hara in its third part shown through some Arab satellite channels during the month of Ramadan 2008 due to the justifications presented in chapter three.

\section{Definition of terms}

Employment; It comes from the verb employ. It comes in the sense of the position or service assigned. Employ means a function for each day. Employment means approval, support, and syndication)). Barth, defines it as an action that is referred to as opening or closing as a result of the succession. The function of language in 
narrative cinema means the character's act whose significance is determined in casting the weave. The procedural definition of employment is that it is the process of borrowing something, quoting, or changing its original components, such as a term, concept, method, process, or other, and inserting it in a new format or within the bounding of a different expressive medium so that it acquires a new appearance or new meaning that it did not previously have when it was in its original field .

\section{Section one}

Significance as a term and as a concept.

Before going into the details of the present study, it is necessary to briefly refer to the term semantics and what it means, its derivations and mechanisms for using it within its original cognitive field or when employed within the structure of neighboring cognitive fields. Al-Muheet dictionary defines it as taken from the verb signified, which means guided. It is said: He guided him to the path. Significance" means guidance and evidence.

In his book General Linguistics, Saussure defines it as the science that studies the signs and symbols of life within social life within the language system. The significance may be related to some extent to the sense that the work maker wants to communicate to the audience. It is a mental image about the details and things that exist in The outside world despite being called by a symbolic or verbal symbol. It is related to the issue of producing meaning through joint and mutual communication between the sender and the receiver or what the sender wants to communicate to the recipient.

Significance is the ability to perceive something due to the presence of something inherent to it. For example, if one hears the doorbell, automatically, his/her mind turns to a conclusion that someone is at the door waiting for permission to enter or inquire about something. This process of mental transmission has its foundations in the memory of the person. Usually, it is a mental correlation between the sound of the bell and the presence of someone at the door. According to Saussure's theory, signifier is the sound of the outside bell, but the signified is the presence of a person waiting at the door. The cinema could quote many concepts, terminology, and techniques of linguistic origins and employ them within the expressive medium, which is represented in the visual image. One of the researchers states that the multiplicity of significance and ambiguity must be seen as factions of virtues. The tension indicated intentionally between meanings reveals a lot about the nature of the visual discourse. Accordingly, the researcher establishes a procedural definition of the significance to be used in the present study. Significance is the value of the effect and the meaning that the text or discourse leaves in the recipient as a result of exposure to that thing or interacting with it, whether it is positive or negative, so as to expand the area of the meaning emanating from it and multiply its interpretations. Significance is either verbal or non-verbal as in the figure below.

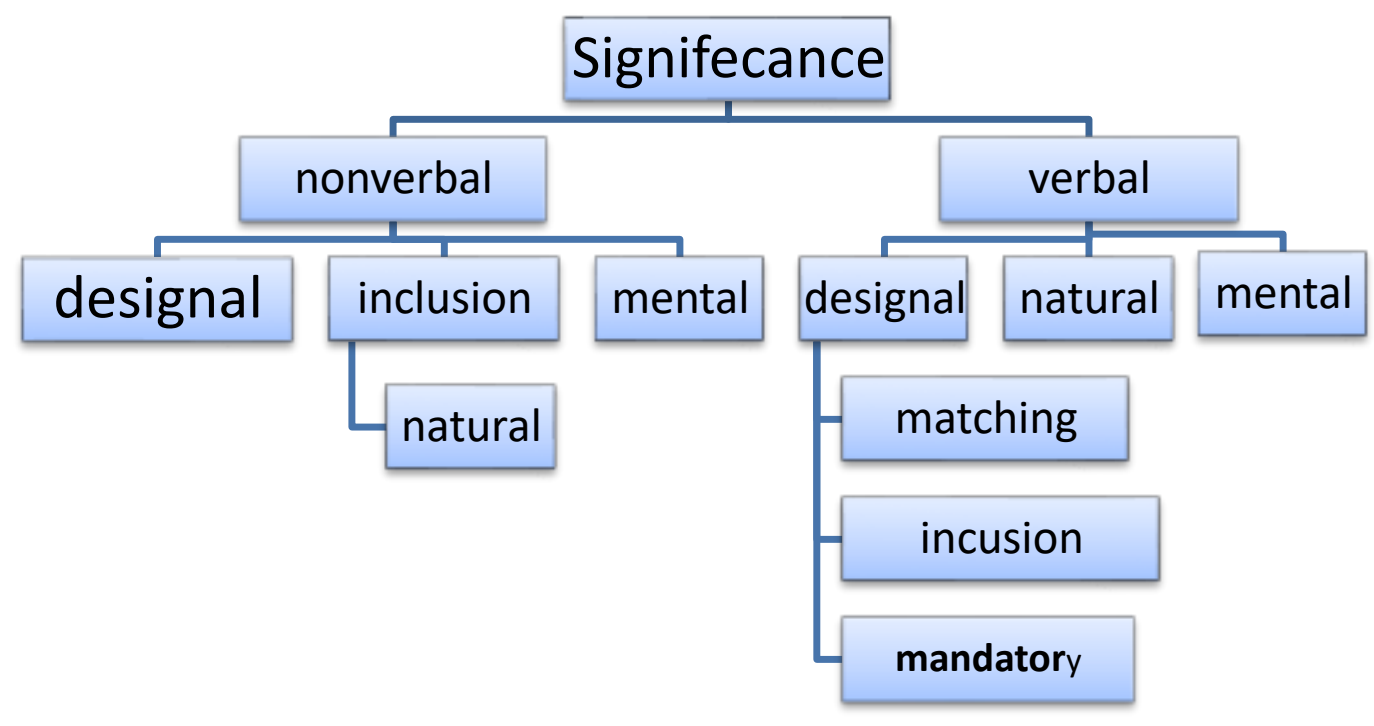

In this figure, significance is so much used in the language of art in general and the field of visual or mental images in particular. There are many opinions about it to the extent that it almost lost its original 
meaning. The reason for this problem is its replacive feature and its superior flexibility on employment or use within Any specific linguistic or pictorial context. For example, it is often said that an artwork has such and such significance and these pictures indicate such and such.

The prevailing belief was that the topic of significance and its sub-finishing is an issue outside the scope of the cognitive and scientific investigation. That can be observed in some recent scientific studies that may confirm or try to highlight that the subject of significance is an external matter and the artistic discourse does not have a close relationship with it. But, after the emergence of studies and philosophical and aesthetic trends with a very precise specialization, the subject of significance received a great deal of importance.

Barthes states that this type of studies gives a meaning that there is no meaning. So, its significance is meaningless. Significance may be a floating and elastic concept that is not subject to accurate numerical analyzes. The researcher tries to illuminate something from this topic and tries to solve some ambiguities in this subject. Hazim Al-Qartajani states that significance is the image that comes to mind About the things.

Ibn Sina states that significance is that if an imagination is drawn, its meaning is ordained in the soul, then the soul knows that this audible is for this concept. Whenever the sense of the soul is mentioned, the soul turns to its meaning. Significance is the salvation of the expressive work of the visual or linguistic discourse. It emerges and acquires a mechanism of interrogation that transcends the classic viewing of visual discourse. It is a continuous search, diligence, mental, and intellectual achievement in the internal structures of the image and the modalities of its operation. The difference of recipients in the mental image is purely subjective and not general, comprehensive, and common with other recipients despite the fact that it sometimes involves common characteristics. Two different elements can be distinguished:

1. Signifier: It is the thing in itself. This is what poetry and literature critics go to.

2. Signified or sign: It is the personal relationship with the mental thing or phenomenon in which this thing is understood. This is what linguists go to.

The researcher indicates that the above quote tries to indicate the nature of the external and internal relations between the work and its recipients. There are also two different ways to understand the significance of the discourse according to one of the researchers:

1. Interpretation of the internal structure. It is the semiotic approach of Vincent following the structural school.

2. Tracking the various relations of discourse. Understanding the discourse is not a matter of searching for a hidden intention behind it. It rather follows its movement of meaning and significance towards the indicative world. Or, it follows interpretation in a world that is open to discourse, which is the method of following the aesthetics of receiving.

The researcher points out that there is a difference between significance and interpretation. He prefers not to confuse them with each other because they may be used at several times. Many authors use them as if they have a single intent or something of similar significance, which is generated by the visual discourse as a result of the movement of visualizations and assets within the image. The discourse contains ideas that are not visible, but they are mental. They may be clearly embodied by the image exclusively.

It is evident to say that significance is not fully and completely understood except within a specific pictorial format, that is, within a set of consecutive images within a scenic scene indicating a specific meaning. Significance does not disclose itself sometimes except within the context and its meaning may not be completed sometimes except within the integrated system of the visual discourse in some Great philosophical works. A single image possesses meanings that are dense and deep and may not be carried by themselves or the scene alone. So, any significance is only realized within the overall structure of the visual discourse. The context or format may determine the significance of the image through three main pillars:

1. The what of the image; The context determines which image is displayed during the scene and defines its direct meaning and what it aims on its own.

2. Defining the idea; What idea the image expresses and how it serves the scenic layout.

3. Rough completion; The context determines that the full and complete idea of the scene has been presented and its meaning has been explained.

Thus, without the layout or context, the recipient does not understand the meaning of the image, especially with unawareness of the phenomenon of anagrams or the multiplicity of connotations that may accompany a visual image.

Some people may ask, is it necessary to have special rules and principles in order to be able to form, or to produce a specific significance that is sent to the recipient with the aim of influencing it, whether it is a positive or negative way? In other words, is it possible to capture the ordinary recipients by significance and 
its ramifications within the image space through chaos or Lack of internal installations of visuals and picture assets? The answer is yes. Dudley Andrew states that everything in cinema has a second meaning. The researcher emphasizes that this meaning or significance may be a somewhat chaotic ramification that may be understood far from the directing intent of the maker. For example, if the film had been dominated by hot colors, especially red, the audience would have thought that there was a Marxist or revolutionary tendency within the movie's structure even though the director might not want that. These meanings appear as follows: 1. Replacement relationships; These are the relationships that arise between members of one group (single, spinster).

2. Integrative Relationships; These are the relationships that arise typically and not necessarily between different expressions (unmarried / man-woman).

Replacement relations are also divided into the following:

1. Inclusion relationships; It is represented by an expression like (dog / animal). The first one is an inclusion in relation to the second. This means that the significance of a dog includes the indication of an animal.

2. Relationships of disharmony are represented by two expressions. The first relationship has no linkage or intercourse with the second as it is incompatible at some level such as (white / red). For example, a white shirt is not red, and vice versa is also true.

Based on that, the researcher believes that the receiver or TV viewer should know or try to capture the simple and profound connotations that the visual message sends in order to reach an understanding of the contexts, relationships, and functions of all the details, visuals, and characters within the movie. Significance has an external and internal level exchanging roles in light of importance and significance in presence and absence. It is worth noting that one of them may illuminate the other and denote it, and vice versa. Significance may move between their multiple levels to increase the influence and expressive energy of the visual image.

\section{Section two}

\section{Color in TV}

Linguistically, color refers to the form; blackness, redness, and so on. Color is defined as the color of everything that separates it from others. It represents the identity of the object, whether it is a plant or an inanimate object.

Physically, color is the effect resulting from the interaction of light with the printed surface and its reflection on the eye retina. The sense of color and its mental perception is according to the experience of the recipient. Chemically, color is defined as a group of complex reactions. This concept revolves around the composition and placement of colors.

Arts exploited color in various branches and absorbed it in different fields. In artistic works, it intertwines with most of the joints of artistic works and their structural elements whether it is a twodimensional or three-dimensional art.

As for the seventh art; cinema, color is cinematic in multiple ways and mechanisms according to the content of the cinematography and the way it is directed. Color is a final indication of the process of analyzing white color through a prism that begins in red and ends in violet, passing through orange, yellow, green, blue, and indigo.

The sunlight is composed of a mixture of different colors. Dropping this light through a light incision on one side of the triple prism of the glass and receiving the prominent rays from it on a white board will dissipate or dissolve the light into a group of colors called the solar spectrum.

The American scientist Vernet states that the primary colors are red, blue, and green. These colors result if lights of red, green, and blue are mixed in the correct proportions. White color results when the three lights meet. But, when two lights meet, the resulting light is the established color with the third primary color. All colors found in nature can be obtained by mixing two of these colors.

The other three colors, which are green, orange, and violet are called binary colors produced by mixing two primary colors.

Green results from mixing blue and yellow.

Orange results from mixing red with yellow.

Violet results from mixing red and blue. 
Cold colors are the colors confined to the spectrum between greenish yellow and violet, which is the coldest color. Blue is a cold color because water and sky are the source of coldness. Greenish color can be considered a cold color if a certain amount of its yellowness is deleted.

Hot colors are the colors that are confined to the spectrum between (greenish yellow and red which is the hottest color. Greenish yellow color can be considered a hot color if a certain amount of yellowness is added to it. Red and yellow are warm colors because fire, sun, and blood are sources of heat and light.

The color degree is measured by considering that each color has a scale that ranges from white to intense or close to white. By that scale, the color consists of six grades that can also be doubled.

Compatibility means colors that relax the nerves in the eye.

Looking at red and orange reveals that they are compatible as well as light and dark blue are compatible. Each two colors adjacent to the spectrum are compatible.

If the yellow and red are combined, the compatibility appears. Also, red and blue result in a purple color. Yellow and blue result in green.

Green and orange can be used with green, purple, green with blue, orange, purple with red, yellow and red with brown.

Bright colors have a strong impact on the eye.

In life, colors are related to the meanings in the subconscious as a result of experiences, some of which are inherited, and the other are experienced in life. Colors, as a visible thing, increase stability and graphics in minds than any experiences gained through other senses.

It is easy to remember color than to remember a voice. Hence, colors have certain meanings and a correlation with the circumstances and events. This is a solution to the reasons that cause some people to tend to use some colors rather than others.

Experiments and psychological tests conducted on a group of individuals who differ in their inclinations and cultures have led to general indications of colors among people.

\section{Indications of some colors}

1. Black color is associated with death, fear, sadness, blindness, and night.

2. White color is associated with purity and hygiene.

3. Red color is related to fire, flames, heat, warmth, blood, and killing. It raises nerves. Many people do not feel comfortable for it. Because of the association with warmth, it has a role in sexual thinking.

4. Green color is associated with gardens and trees. It is associated with calm nerves. It indicates the meaning of blessing and heaven.

5. Yellow color is associated with the sun, light, jealousy, and hatred.

6. Blue color is related to the sky and water in nature. It is an appropriate role for calmness and coldness at night. If blue meets green, the result is coldness.

In cinema and television, color includes a lot of connotations and meanings because color is a visual and not verbal subject. It moves between the physical and down to the aesthetic and semantic employment for it because it carries intellectual implications because it contains a high energy of ignition as a result of what the human soul stores of psychological and social implications related to color and the external shape For any artistic achievement. Color is a complex subject and it does not affect the ability to distinguish between things only, it also changes the mood and feelings and affects preferences and aesthetic experience in a way that exceeds the influence of any other element that depends on the sense of sight or any other sense.

Color affects the recipient as the first one to contact with it from the sum of the items of the artwork. Then, the sensory perception process that succeeds certain responses occurs according to the stimuli that color emits and signals that nurture the recipient's mind and push it to explore the contents of the artwork. Color has three implications:

1. The color feature; It is the origin of the color that distinguishes one color from another. It can change the origin of the color when mixed with another color.

2. The value; It is the degree which the color has, such as being light or dark.

3. Saturation; It is the feature that indicates the purity of the color to the extent of its saturation. It is related to the extent of its selection or the extent of its mixing with black, white, and gray. There are three cases of lack of saturation:

A. Lack of saturation due to the mixing of the origin of the color with an amount of white. In this case, it is said that the origin of the color has been diluted. 
B- Lack of saturation due to the mixing of the origin of the color with an amount of black, in which case, it is said that the origin of the color has shaded or has become darker.

C. Lack of saturation due to the mixing of the origin of the color with a percentage of gray color. In this case, it is said that the origin of the color has neutralized,, which means that it became neutral or darker.

The artist is supposed to deal with these phenomena and properties with complete seriousness because they operate at internal, external, or subjective or objective levels. The first means the related effects in the recipient itself, such as previous experiences and realistic references. The external is through the general form that includes color as well as The content within that shape. Cézanne asserts that when the color possesses its richness ..., the form possesses its integrity.

The difference between the subjective and the objective meaning.

The difference between the intention of the author; the work maker and the intention of the reader; the recipient of the work)) directly agrees with the role of personal identity for the manufacturer and the recipient in terms of transmitting and receiving in searching for the basic meaning of the artwork. The color has many indications. Researchers disagreed about it in some joints, and they agreed mostly and more generally, because it has many aesthetic, expressive, symbolic, and psychological functions and indications, as well as the most important and larger function and significance, which is the dramatic function. Color is related to the system of other graphic elements within One mass or as an independent element in itself because it possesses high energy in single work to generate new connotations and meanings for visual images and the dramatic scene. It may establish its own meaning added to the special meaning. It may be given to the meaning of the image and determine the meaning of the color itself. It can be said that to use color expressively is as important as the actor or any component of production as the medium for developing expression.

Within its folds, color includes vital energies that help to stimulate the dramatic act and push it forward either by the characters or by the story and the intertwining of its events or by the formative elements that the color enters into its details. It is a psychological experience based on a scientific and physiological principle. Color can change feelings and sensations in taking Attitudes, whether negative or positive, or against dramatic characters, or the position of conflict that takes place on the screen and often uses color not as an aesthetic dye, but rather as indications and symbols that give a wider space to receive the shot and the scene more effectively.

Color is used in cinema and television with multiple mechanisms. According to schools and artistic schools, it is well known that the Impressionist School has employed color in ways that differ radically from what is common in daily life in order to create large shifts in the meaning that emerges from the image because the uses of color originally In the cinema are to add a realism feature to the photo.

Despite this variation in employment, there are currents, doctrines, films and directors who worked in the central region, which connects between realism and impressionism, trying to exploit the so-called ("color incident"), which is a color necessity required by the scene regardless of the sect or current that The film belongs to it and the origin of color is that there is a recipient who wants to enjoy the successive visual image seen between what is realistic in nature and what is fictional in the movie.

The researcher refers to the aesthetic values that recipients experience when they are exposed to a fictional color regardless of their authority, culture, and viewing conditions depending on subjective data related to the recipient and aesthetic gained experiences, whether from visual observations or from life experiences. The aesthetic feeling and artistic taste of color may also depend on objective psychological foundations related to the characteristics of the existing visuals consisting of a bundle of colors that the director installs and sends towards the recipient for the greatest possible effect because color) is an influential structural element that increases the area of functions affecting Viewer knowledge systems.

It should be pointed out that there is a clear difference in the employment of color between cinema and television. The reason for that difference is due to a number of justifications and reasons. Some of them are technical, as the techniques used in cinema are different from those used in television where Variation in the types of cameras used, the nature of their internal components, the mechanisms of their work, and the subsequent printing and acidification process and other mechanisms used in the display, as well as the nature of the raw material used in photography and also in the nature of the use of lighting between cinema and television As well as the nature of the screen used in the presentation in terms of quality and size in addition to the original difference in the style of cinematic work from television. Cinema tends to the philosophical and expressive aspects more than television to some extent due to the nature of the difference of viewers and the receiving conditions. There is another matter which is the nature of production in each of them where there 
is a large and huge costs in film production compared to the capabilities of television. The researcher prefers to warn that these factors combined have changed and to a large extent $m$ Between the last century and the current century as cinema has approached production in a television manner to reduce production costs and to keep abreast of major technological developments as well as the entry of new companies in the competition relying heavily on television production. At the same time, the great tendency of TV producers to match Massive cinematic production should not be forgotten because of its great impact on viewers. There is an increase in the area of the TV screen, even to almost reach proportions close to the cinematic screen. All of these factors have a significant impact on the color position, purity, employment, and its expressive, dramatic, psychological, and aesthetic indications sent towards the viewer in order to influence it and thus achieve A type of interactive communication to serve desired ends.

\section{Section three}

Significance and meaning in the visual employment of color.

Colors possess great connotations. The work maker can employ them in a variety of ways according to his/her directing intent because it has the ability to comply and employ easily, being a real donor first, and secondly, because it has significant gestural implications in the viewer's mindset. In addition to those technical and dramatic aspects of employment and significance, it has its presence and great importance stemming from its great influence in the daily life. The evidence is that there are some people with color blindness whose response and information are sometimes disturbed. It is also used in prisons, solitary prisons, and psychological torture rooms where colors of high wavelengths or warm colors are used because they provoke the nerves of the accused, such as red and orange, which leads to collapse and then confess sometimes. It is also used abundantly in cities of games, kindergartens, and tourist places. Colors may be affected by their meanings and connotations sometimes in The contexts and systems in which they are located as in light signals, sports, medical, and scientific uses.

Thus, directors focus a lot on the subject of color as they enter in most if not all the details of the artwork. Color possesses an escalating energy and interrogative ability by the viewer. It can indicate additional meanings to its original significance. For example, a character inside the movie is exposed to a punch In the eyes resulting in that the shot becomes a black screen only. The black color works as a sign of turning off the light of the eye or a blackness of the outlook on things, visuals, or subsequent events. So, color has inner and External features where color should have a dramatic function and not only a conceptual function. Color is an important element of artistic expression as it may be able to work alone as an independent and significant element. It can also participate in other elements in the formation of new systems. The need for color And its connotations increases whenever the complexity of real or artistic life increases. When the nature of the visual discourse and its philosophical and expressive complexities increase, color helps the increase sympathy and psychological attraction by the viewer to the nature of the film's signals. It is the emotional function of color that emerges from the natural order to establish the color visualization of the action corresponding to the process of forming the living movement of the whole work. The director should take into account the accuracy in employing color and give significance, because using it extensively and abundantly in the movie without a reasonable reason may lead to a kind of misunderstanding or confusion in the collection of its intended significance. It may refer the viewer to other different meanings from what is meant by the director. Color should not attract attention at all unless it has significance and importance necessary for a more complete presentation, detection and demonstration of significance. It is axiomatic to say that colors have their socially recognized significance. Therefore, it is sometimes difficult for the director to try to violate those agreements and details that the audience viewers are accustomed to. Pessimistic characters are made to wear dark colors as they look at things, visuals and other film characters and see them all in one faded color without psychological influence on others, to indicate their illness and their inability to communicate. It is strange that there are colors that may cause complexity or inconvenience to some viewers, even when they are included as a natural element in a normal cinematic or television picture, such as red, for example, which carries with it ideological and political connotations and indicates the sensitivity and resentment of Europeans, Westerners and Americans when entering a context indicating, for example, a political issue or even not political. It denotes the former Soviet socialist system or propaganda of Marxist Communism.

Colors are related to the actual drama. That is indicated by a clear indication. In the movie of (AlRisala) by the director Mustafa Al-Akkad, when the masters of Quraish were mastering the dramatic act and influencing the movement of the dramatic act, colors are bright, strong, and of high energy. But, those colors 
disperse, decay, and deplete until they reach the gray level when they lose control of events and Muslims become more dominant.

Color can actually embrace the drama, prepare for it, and reveal some of its characteristics. For example, in the movie of Salah Al-Din Al-Ayyubi by the director Najdat Ismail Anzur, Arab Muslim leaders wear quiet clothes close to the cold colors area such as blue, green, and white, which indicates the greatness, dignity, and Tolerance. The evidence is what happened after their victory. They showed great mercy and compassion for the defeated army and its leaders and the Frankish people. Hence, color played a role in revealing human nature. Unlike the leaders and some of the Frankish soldiers who wore clothes close to the hot colors like red, yellow, and orange. It must be pointed out that the designation of colors as hot or spread in space was the result of their derivation from hot natural phenomena such as the sun, fire, volcanoes, and others. Cold colors may be due to their derivation from calm natural phenomena such as water, plantations, snow, etc., as well as the nature of colors and their implications and employment may be due to social and psychological aspects related to the collective subconscious or the popular memory of people. This connection is a result of the culture of people and the nature of human and scientific knowledge. Therefore, it sometimes changes with the change of these conditions and data. Accordingly, this necessarily changes the pattern of employment and implications within the structure of work. The artistic work, whether cinematic or television, is affected by these factors because the director is definitely from that environment and that society and is affected by those influences such as traditions, customs, norms, social and cultural norms, as well as the difference between the significance of colors and their uses during a certain period of time.

\section{Theoretical framework indicators}

1. Color differs in its significance of employment according to the culture and traditions of the community.

2. Color cannot be an indicative value or an expression in itself except by working with other elements within the elements of the image.

3. The color value cannot be determined in isolation from light formation as color is an attribute of light at a specific frequency.

4. Color is employed in a variety of ways, depending on the intention of the director or the creator of the work.

5. Color may be accounted for as a result of employing it intentionally, externally, on the meaning and significance of the entire image due to its high operating energy and psychological effects in the scenes.

6. Color can contribute to shaping the image space by creating the illusion and deceiving consideration of building a TV picture.

\section{Chapter three}

The research procedures.

First; The approach; The researcher relied on the descriptive approach, which depends on the analysis because it is appropriate for the nature of the present study.

Second; The research sample; The researcher chose the movie of Bab Al-Hara in its third part shown in 2008 from Arab satellite channels for the following justifications:

1. The distinctive use and expressive employment of the color element in most of the scenes and footage of the movie and the use of modern technologies so that it becomes a sample that can be analyzed accurately.

2. The large proportion of following-up Arab and Iraqi writers, critics, and specialists.

3. The high audience and viewership according to what was reported about it in newspapers, magazines, and TV interviews during its presentation in Ramadan 2008 which was more than the movies shown at the time.

4. The work director is one of the distinguished and award-winning directors.

5. The directing processing of the movie is close to the cinematic processing since the director has a cinematic background.

\section{The sample analysis}

The movie name; Bab Al Hara Part Three

Directed by; Bassam Al-Mulla.

Performing; Samer Al-Masry, Sabah Al-Jazaery, Saleem Klass, Jumana Murad, Wael Sharaf, and Wafa Musli. 
Production; Al-Aaj company.

Shown on the MBC satellite channel during Ramadan, as well as on some Arab satellite channels, such as the Libyan, Bahraini, Omani channels, and others.

The story of the movie talks about a popular Syrian neighborhood in Damascus and some of the surrounding areas during the period of the French occupation in the twenties of the last century through the traditional social relations between the residents of this neighborhood and the other neighborhoods adjacent to it and tensions and clashes that interspersed this relationship. But, at the end, it reaches the inevitable conclusion, which is the necessity for everyone to stand together against the enemy, whether internal or external.

\section{The analysis}

In the first episode, specifically in the first scene, the first uses and indicative uses of color are embodied in the Bab Al-Hara movie, where a group of colors, a set of dark colors that send sadness and pessimism in the psyche of the viewer are noticed. This indication becomes clearer with the absence of any dialogue between dramatic characters despite their visual presence that is embodied on the screen in relatively large numbers. Most of the characters are men attending what looks like a funeral. The viewer does not know the reason for this attendance and this sad occasion, especially it is the first scene of the first episode in the movie. The appropriate use of music and sound effects give The indications of sadness. This mixing between the voice stream and the colors has provided the appropriate ground for transmitting intellectual messages with multiple connotations towards the recipient of the movie. Then, the recipient discovers the absence of an important and main character in the movie that has a distinguished presence in its second part, which was previously shown in the last year, which is the character of Abu Essam who is considered one of the most prominent characters due to the influence in the course of events, in the dramatic presence, and also in the dominant visual dramatic power of the atmosphere of the scene in which it participates. For example, the black color is a social customary indication of sadness, death, pessimism, and lack of life. Therefore, using it in this scene gives this color its significant and influential indications, whether in the nature of events or in the process of receiving the movie. When the director employed the black color in this way, he gave it a great semantic value and a high exploitative energy so that it acquired most of the indications and meanings of the scene, especially with the intended absence by the director or the scenario for the dialogue element that usually reveals the nature of the scene Or the snapshot or the nature of the story narrated on the screen. Lighting helped by its distinctive employment in this scene as it was somewhat dim to add a kind of realism to the scene. The process of mating and interaction between colors and lighting was very harmonious and coherent as it gave large and deep meaning to the recipient. The researcher claims that they have been able to communicate the value, meaning, and significance of the scene to the audience.

Accordingly, it is possible to confirm that color has the power to imply meaning or express it directly according to the intentions of the director or depending on the recipient's culture and its intellectual and social references as well as its observations and experience in receiving cinematic or television works.

As for the seventeenth episode, in the scene in which the Sheikh of the mosque comes out after performing the Maghrib prayer and Essam comes to him in order to persuade him to go to return his wife Lutfia who has been staying for some time in her mother's house because she had a problem with Essam's mother, the semantic expressive employment of colors, especially the light blue color is a sign by the director to denote the atmosphere of faith that surrounds the space of the mosque or to suggest the atmosphere of faith that is around the works of the Sheikh of the Mosque, who is seen as a beloved and kind-hearted figure who seeks to serve the people of the neighborhood. Light green, white, and some colors with short wavelengths derive their colors from cold and calm natural colors, such as sky and sea. This employment gives great indications to the recipient and affects a lot of psychology due to the nature of these colors and their impact on the recipient's nerves,. The result of their realistic connection in Everyday life makes it possible to say that these colors usually accompany the good and quiet characters in most films. Therefore, the recipient can judge that the sheikh of the mosque will succeed in the process of returning the wife Lutfia from her mother's house despite the latter's insistence not to return. This is what actually happened and the wife returned despite the multiple attempts.

In the seventh episode, specifically in the scene of the Meeting of the women of the neighborhood at the house of Umm Issam, the camera displays the assets of the house inside, especially those configurations, accessories, furniture and decorations in the courtyard of the house. In the center of the house, there is a large water basin that looks like a fountain surrounded by cushions of plantations of joyful colors that start 
from light colors until they reach dark colors. The colors are expressively employed in order to give expressive indications of the love, kindness, cleanliness, goodness, tolerance, and other characteristics that make The person loved by others so that it is popularly said to the good cooperating person that he/she resembles a fragrant rose that radiates a perfume on others. The director employed these colors in this scene to give certain connotations in cooperation with the other color system included in the composition of the scene as well as with the system of other visual and sound elements Where the colors interact with the sound of flowing calm water, which inspires reassurance, harmony, and peace. Thus, in addition to the quality of the music and sound effects that take place in this scene, the interaction of these elements may form an additional meaning to the original meaning that emerges from the story or tale that is narrated both formally and verbally through dialogue.

In some of the episodes in which the Al-Aqeed character, which is brilliantly performed by the actor Samir Al-Masri, specifically in the scenes that took place in his office or workplace, there is a meaningful employment expressing color. It reveals the nature of the character that lives or works in this place. The colors of the decorations involve purple or brown. They start from light beige to dark brown that looks like coffee. This gives great expressions and indications as it may symbolize the color of the mountain, the highlands, and the colors of the rocks that are characterized by cruelty, lofty, and difficulty to break. That is equivalent to objective and pictorial With the dramatic character performed by the Al-Aqeed because he was responsible for the security of the neighborhood, its problems, and its internal and external conflicts with other lanes as well as his hidden and declared conflict with the French occupation and its spies. This gives strong and influential indications of the cohesion of this character and the strength of its dramatic or psychological construction. This enhances the dramatic act of Al-Aqeed and gives him additional connotations, which gives the recipient a sense of relief to the fate of the neighborhood. This is manifested in the conflict of Al-Aqeed with the police officer called Abu Jawdat. This employment expressed by the colormaker in these scenes gives indications of how to create a space for the character through the colors and configurations surrounding it and giving it additional dimensions that establish important relationships with the recipient to communicate positively with the scenes and clips in which this dramatic character appears. This establishment is necessary to create sympathy for the character and the movie in general.

In the twenty-fifth episode, specifically in the scene in which the soldier Samaou searches for the son of the killer of his father in the farms near the outskirts of the neighborhood, they finally meet near one of the fruitful trees. The work maker employed a beautiful and expressive color in synergy with other elements, especially the element of lighting. There are three groups of color and lighting configurations. These configurations have contributed to highlighting the depth of the image and giving it multiple levels of depth as a result of the appropriate employment of colors with the help of visual configurations. The first of these color configurations is the soldier Samaou's color as he changes his military clothes For the first time in the movie. He wears the classic Levantine outfit represented by the wide black trousers and a light-colored shirt to indicate in advance what will happen to the dramatic event and the fate of the character of the son of his father's alleged killer. They meet. He holds a pistol in his hand that he previously stole from the police chief called Abu Jawdat, but Samaou pardons the son of his father's killer to signify his eminence and his noble Syrian kindness. The researcher believes that the colors have contributed to a large extent to the prior detection as a result of not wearing strict military uniforms. In another corner of the shot or other shots of the scene, there are appropriate uses of blue to indicate the spiritual atmosphere that prevails in the scene as well as dust or steam Or the white smoke rising in the background of the scene to give expressive indications of the escalation of anger at Samaou from the son of his father's killer. The black color that the son of the killer wore as a cover for the head may give an indication of sadness and sorrow in which he cures grief over the painful and unfortunate incident caused by his father as a result of his unintentional or alleged killing of the father of Samaou. It may give additional indications of his grief over his leaving the French officer who took him captive, who may be defeated if Samaou kills him. Thus, the revolt of the people of the neighborhood of the French is lost, but the maker of the work could surprise the recipient by the development of events later when Samaou pardoned the son of the killer of his father. Thus, this appropriate employment of colors and lighting helped to activate the dramatic act and predict it in advance. This is what makes the recipient always surprised by the events as the recipient's knowledge of the progress of the story and the events in any movie may lead to the departure from pursuing that artwork for having revealed the dramatic game or having known it in advance, which leads to the lack of influence of the movie on the hearts of the viewers. Colors, the nature of their physical composition, and knowledge of their social effects in viewers 'memories can, with 
appropriate and expressive employment and function, increase the area of suspense and excitement to the dramatic story narrated by pictures and dialogue.

In the closing scene of the third part, which revolves near the outer door of the neighborhood, there is a distinctive use of colors as they worked with their maximum expressive energy to indicate ideas and data that may be present or absent in the image space. The men of the neighborhood have lined up behind the closed door in the form of two walls. One of them is Hidden. The second is visible. These two levels were formed as a result of the director's vision and the scriptwriter's craft in order to create a final surprise that concludes the movie in this part with the hope of returning next Ramadan in the fourth part of the movie. Colors, costumes, and characters overlap with each other To give indications of strength, alignment, and unity of destiny that awaits the brave men lining up behind the door of their neighborhood. This indicates the strength, solidity, aggression, and alignment. This color overlap reveals the nature of the characters. It may send indications to the recipient that all social categories within the fabric of the neighborhood unite together against the aggressor or whoever wants to harm their dignity and pride of their neighborhood. This expressive employment of colors by the maker of the work increases the flow of meanings and ideas and gives a large space for interpretation. So, it does not capture only one meaning, but several levels of meanings. This is what is required from TV works with good specifications that have to affect The recipient.

\section{Chapter four}

\section{The results}

1. Color employments are subject to the popular taste associated with the community environment in which the events of the movie take place, the quality of the viewers and their cultures, and their awareness of the significance of colors.

2. Colors in the picture work in synergy with each other to produce specific connotations that the viewer analyzes with various methods. This interplay between the elements is what gives the image its implications.

3. There is a correlation between color and light in the image as one cannot be separated from the other for physical and technical reasons. Thus, the production of signification may be shared between them.

4. The nature of employment of colors in the image varies according to the director's style and the nature of the written scenario, as well as the nature of the color itself.

5. Color is the most prevalent and dominant element in the image as it is included in the structure of all other elements.

6. When using it in a certain way, color contributes to creating a sense of depth, illusions and visual illusions in the space of the visual image.

\section{CONCLUSIONS}

1. Colors are not decorative elements added to the movie, but they dramatic, aesthetic, and expressive needs depending on the director's philosophy and his/her style, as well as on the recipient's references.

2. In addition to the nature of the color itself, it is often included with the multi-color group to give additional significance to the movie.

3. The physical nature of color necessitates it to participate and interfere with when lighting to form a unified meaning. It may not be possible to separate them due to the nature of the overlap between them.

4. Color, as being included in the formation of any visible detail in the image, spreads and extends the image space significantly.

\section{REFERENCES}

The Glorious Quran.

Andrew, D. (1987). Major Film Theories, Translated by: Gerges Fouad Al-Rashidi, Egyptian Book Authority, Cairo.

Al-Andalusi, Abu al-Hassan Ali bin Ismail, (1987). Dar al-Fikr, Beirut.

Eisenstein, Sergey, Film Director's Notes, Translated by: Anwar Al-Bishri, Egypt, Egyptian Public Foundation for Authorship, Printing and Publishing, 19. 
Pavis, P. (1992). Stage Languages, Translated by: Ahmed Abdulfattah, Ministry of Culture, Cairo.

Punta, J. (1996). Architecture and Its Interpretation, Translated by: Souad Abd Ali, House of Cultural Affairs, Baghdad.

Hussein, Q. (1982). The Psychology of Perception of Color and Shape, Dar Al-Rasheed for Printing, Baghdad.

Al-Badawi, K. (2000). How to Learn and Teach Color, Amman, Dar Al-Isra.

Al-Razi, M. (1993). Mukhtar Al-Sahah, Dar Al-Risala, Kuwait.

Radhi, M. (2005). The Art of Light, Damascus, The General Cinema Foundation.

Riadh, A. (1965). Color Photography, Egyptian Biblical Library, Cairo, First Printing Press.

Reid, H. (1983). The Present of Art, Translated by: Samir Ali, House of Cultural Affairs, Baghdad.

Salim, A. (1986). Cinematography, Filmography, House of Cultural Affairs, Baghdad.

Stolentis, J. (1974). Art Criticism, Translated by: Zakaria Ibrahim, Ain Shams Press, Cairo.

Alloush, S. (1983). Glossary of Contemporary Literary Terms, Beirut.

Shamil, A. (1992). Color: Theory and Application, Studies in Art of Architecture, Baghdad.

Al-Dhahir, A. (1980).Arranging Al-Muheet dictionary on the way of the enlightening lamp and the basis for rhetoric, part four, Dar Al-Arabiya Book, 3rd edition, Bab Al-Waw.

Fadhl, S. (1987). The Structural Theory in the Arabic Criticism, House of cultural affairs, Baghdad.

Al-Qadhi, A. (1983). Arab Science of Evidence, Contemporary Arab Thought, National Development Center, Beirut, No. 18-19.

Al-Qartajani, H. (1981). The Methods of Rhetoric and Authors lamb, 2nd Edition, Dar Al-Arab Islamic, Beirut. Qalaj, S. (1975). Aesthetics of Color in Cinema, Egypt.

Lines, J. (1986). Language, Meaning, and Context, Translated by: Abbas Sadiq Al-Wakeel, House of Cultural Affairs, Baghdad.

Lowe, G. (1981). Understanding Cinema, Translated by: Jafar Ali, Dar Al-Rasheed for Printing, Baghdad.

Martin, M. (1974). Cinematic Language, Translated by: Souad Makkawi, The Egyptian Book Organization, Cairo.

Muhammad, R. (1973). Al-Misfar: An Introduction to Logic, Al-Najaf Press.

Al-Mudarriss, H. (1990). Screen Drama, Part Two, The Egyptian Book House, Cairo.

Al-Waseet Glossary, Part One, Dar Al-Ihya for Arab Heritage, Beirut, Bab Al-Dal.

Al-Waseet Glossary, Part Two, Dar Al-Ihya for Arab Heritage, Beirut, Bab Al-Waw.

Hooks, T. (1987). Structuralism and Signal Science, Translated by: Majeed Al-Mashta, Baghdad.

Yousif, S. (1990). Language Psychology and Mental Illness, Knowledge World Series, National Council for Culture and Arts, Kuwait.

Duraid, S. (1999). Kinematics of Time Structure Systems in feature film, a PhD dissertation, University of Baghdad, Faculty of Fine Arts. 